\title{
Periodic solutions for a kind of neutral functional differential systems
}

\section{Zhi Min $\mathrm{He}^{1 *}$ and Bo Du²}

${ }^{*}$ Correspondence:

hzm2007@aliyun.com

'Tianmu College, Zhejiang A and $\mathrm{F}$

University, Hangzhou, China

Full list of author information is

available at the end of the article

\begin{abstract}
In this paper, we analyze some properties of the linear difference operator $A: C_{T} \rightarrow C_{T},[A x](t)=x(t)-V(t) x(t-\tau)$, and then, by using the coincidence degree theory of Mawhin, a kind of neutral differential systems with non-constant matrix is studied. Some new results on the existence of periodicity are obtained. It is worth noting that $V(t)$ is no longer a constant matrix, which is different from the corresponding ones of past work.
\end{abstract}

Keywords: periodic solutions; neutral; Mawhin's continuation theorem

\section{Introduction}

The field of neutral functional equations (in short NFDEs) is making significant breakthroughs in its practice; it is no longer only a specialist's field. In many practical systems, models of systems are described by NFDEs in which the models depend on the delays of state and state derivatives. Practical examples for neutral systems include population ecology, heat exchanges, mechanics, and economics; see [1-4]. In particular, qualitative analysis such as periodicity and stability of solutions of NFDEs has been studied extensively by many authors. We refer to [5-12] for some recent work on the subject of periodicity and stability of neutral equations.

In the last few years, the stability of neutral systems of various classes with time delays has received an ever-growing interest from many authors. Many sufficient conditions have been proposed to guarantee the asymptotic stability for neutral time delay systems. We only mention the work of some authors [13-15]. It is well known that the existence of periodic solutions of neutral equations and neutral systems is a very basic and important problem, which plays a role similar to stability. Thus, it is reasonable to seek conditions under which the resulting periodic neutral system would have a periodic solution. Much progress has been seen in this direction and many criteria are established based on different approaches. However, there is no paper for investigating the existence of periodic solutions of neutral system with non-constant matrix. In addition, to the best of our knowledge, most of the existing results deal with scalar NFEDs or neutral systems with a constant matrix. For example, in papers [16-20], based on Mawhin's continuation theorem, several types of scalar neutral equations have been

(c) $2014 \mathrm{He}$ and Du; licensee Springer. This is an Open Access article distributed under the terms of the Creative Commons Attribution License (http://creativecommons.org/licenses/by/4.0), which permits unrestricted use, distribution, and reproduction in any medium, provided the original work is properly credited. 
studied:

$$
\begin{aligned}
& \frac{d^{2}}{d t^{2}}(u(t)-k u(t-\tau))=f(u(t)) u^{\prime}(t)+\alpha(t) g(u(t))+\sum_{j=1}^{n} \beta_{j}(t) g\left(u\left(t-\gamma_{j}(t)\right)\right)+p(t), \\
& \frac{d N}{d t}=N(t)\left[\alpha(t)-\beta(t) N(t)-\sum_{j=1}^{n} b_{j}(t) N\left(t-\sigma_{j}(t)\right)-\sum_{i=1}^{m} c_{i}(t) N^{\prime}\left(t-\tau_{i}(t)\right)\right], \\
& \frac{d N}{d t}=N(t)\left[r(t)-\sum_{j=1}^{n} \alpha_{j}(t) \ln N\left(t-\sigma_{j}(t)\right)-\sum_{i=1}^{m} b_{i}(t) \frac{d}{d t} \ln \left(t-\tau_{i}(t)\right)\right], \\
& x^{\prime}(t)+\alpha x^{\prime}(t-\tau)=f(t, x(t)), \\
& (u(t)+B u(t-\tau))^{\prime}=g_{1}(t, u(t))-g_{2}\left(t, u\left(t-\tau_{1}\right)\right)+p(t) .
\end{aligned}
$$

For a neutral system, we note that Lu and Ge [21] studied the following system:

$$
\frac{d^{2}}{d t^{2}}(x(t)-C x(t-\widetilde{\tau}))+\frac{d}{d t} \operatorname{grad} F(x(t))+\operatorname{grad} G(x(t-\tau(t)))=p(t) .
$$

But $C$ is a constant symmetric matrix. The purpose of this paper is to investigate the existence of periodic solutions to the nonlinear neutral system with non-constant matrix of the form

$$
\frac{d^{2}}{d t^{2}}(x(t)-C(t) x(t-\tau))+\frac{d}{d t} \operatorname{grad} F(x(t))+\operatorname{grad} G(x(t-\gamma(t)))=p(t),
$$

where $x \in \mathbb{R}^{n}, C(t)=\operatorname{diag}\left(c_{1}(t), c_{2}(t), \ldots, c_{n}(t)\right), C(t+T)=C(t) ; F(x) \in C^{2}\left(\mathbb{R}^{n}, \mathbb{R}\right), G(x) \in$ $C^{1}\left(\mathbb{R}^{n}, \mathbb{R}\right) ; p \in\left(\mathbb{R}, \mathbb{R}^{n}\right), p(t+T)=p(t) ; \gamma \in C(\mathbb{R}, \mathbb{R}), \gamma(t+T)=\gamma(t) ; T$, and $\tau$ are given constants with $T>0$.

Throughout this paper, we use some notation:

(1) $I_{n}=\{1,2, \ldots, n\} ; \forall a=\left(a_{1}, a_{2}, \ldots, a_{n}\right)^{T} \in \mathbb{R}^{n},|a|=\left(\sum_{i=1}^{n}\left|a_{i}\right|^{2}\right)^{\frac{1}{2}}$;

(2) $C_{T}=\left\{x: x \in C\left(\mathbb{R}, \mathbb{R}^{n}\right), x(t+T)=x(t), \forall t \in \mathbb{R}\right\}$ with the norm

$$
|\varphi|_{0}=\max _{t \in[0, T]}|\varphi(t)|, \quad \forall \varphi \in C_{T}
$$

(3) $C_{T}^{1}=\left\{x: x \in C^{1}\left(\mathbb{R}, \mathbb{R}^{n}\right), x(t+T)=x(t), \forall t \in \mathbb{R}\right\}$ with the norm

$$
\|\varphi\|=\max _{t \in[0, T]}\left\{|\varphi|_{0},\left|\varphi^{\prime}\right|_{0}\right\}, \quad \forall \varphi \in C_{T}^{1} .
$$

Clearly, $C_{T}$ and $C_{T}^{1}$ are Banach spaces.

\section{Main lemmas}

Lemma 2.1 [22] If $|c(t)| \neq 1$, then operator $A_{1}$ has a continuous inverse $A_{1}^{-1}$ on $C_{T}$, satisfying

(1) $\quad\left[A_{1}^{-1} f\right](t)= \begin{cases}f(t)+\sum_{j=1}^{\infty} \prod_{i=1}^{j} c(t-(i-1) \tau) f(t-j \tau), & c_{0}<1, \forall f \in C_{T}, \\ -\frac{f(t+\tau)}{c(t+\tau)}-\sum_{j=1}^{\infty} \prod_{i=1}^{j+1} \frac{1}{c(t+i \tau)} f(t+j \tau+\tau), & \sigma>1, \forall f \in C_{T},\end{cases}$ 


$$
\begin{aligned}
& \text { (2) } \int_{0}^{T}\left|\left[A_{1}^{-1} f\right](t)\right| d t \leq \begin{cases}\frac{1}{1-c_{0}} \int_{0}^{T}|f(t)| d t, & c_{0}<1, \forall f \in C_{T}, \\
\frac{1}{\sigma-1} \int_{0}^{T}|f(t)| d t, & \sigma>1, \forall f \in C_{T},\end{cases} \\
& \text { (3) }\left\|A_{1}^{-1} f\right\|_{0} \leq \begin{cases}\frac{|f|_{0}}{1-c_{0}}, & c_{0}<1, \forall f \in C_{T}, \\
\frac{|f| 0_{0}}{\sigma-1}, & \sigma>1, \forall f \in C_{T} .\end{cases}
\end{aligned}
$$

Here

$$
c_{0}=\max _{t \in[0, T]}|c(t)|, \quad \sigma=\min _{t \in[0, T]}|c(t)| .
$$

Let

$$
\mathcal{A}: C_{T} \longrightarrow C_{T}, \quad[\mathcal{A}](t)=x(t)-V(t) x(t-\tau)
$$

where $\forall t \in \mathbb{R}, V(t) \in C_{T}^{1}$ is a real symmetric matrix.

We will give some properties of $\mathcal{A}$.

Lemma 2.2 Suppose that $\lambda_{1}(t), \lambda_{2}(t), \ldots, \lambda_{n}(t)$ are eigenvalues of $V(t)$. Then the operator $\mathcal{A}$ has continuous inverse $\mathcal{A}^{-1}$ on $C_{T}$, satisfying

$$
\begin{aligned}
& \text { (1) } \int_{0}^{T}\left|\left[\mathcal{A}^{-1} f\right](t)\right| d t \leq \begin{cases}\left(\sum_{i=1}^{n} \frac{1}{\left(1-\lambda_{i, L}\right)^{2}}\right)^{\frac{1}{2}} \int_{0}^{T}|f(t)| d t, & \lambda_{i, L}<1, \forall f \in C_{T}, \\
\left(\sum_{i=1}^{n} \frac{1}{\left(1-\lambda_{i, l}\right)^{2}}\right)^{\frac{1}{2}} \int_{0}^{T}|f(t)| d t, & \lambda_{i, l}>1, \forall f \in C_{T},\end{cases} \\
& \text { (2) }\left|\left[\mathcal{A}^{-1} f\right]\right|_{0} \leq \begin{cases}\left(\sum_{i=1}^{n} \frac{1}{\left(1-\lambda_{i, L}\right)^{2}}\right)^{\frac{1}{2}}|f|_{0}, & \lambda_{i, L}<1, \forall f \in C_{T}, \\
\left(\sum_{i=1}^{n} \frac{1}{\left(1-\lambda_{i, l}\right)^{2}}\right)^{\frac{1}{2}}|f|_{0}, & \lambda_{i, l}>1, \forall f \in C_{T},\end{cases}
\end{aligned}
$$

where

$$
\lambda_{i, L}=\max _{t \in[0, T]}\left|\lambda_{i}(t)\right|, \quad \lambda_{i, l}=\min _{t \in[0, T]}\left|\lambda_{i}(t)\right|, \quad i \in I_{n} .
$$

Proof (1) Since $V(t)$ is a real symmetric matrix, there exists an orthogonal matrix $U(t)$ such that

$$
U(t) V(t) U^{T}(t)=E_{\lambda}(t)=\operatorname{diag}\left(\lambda_{1}(t), \lambda_{2}(t), \ldots, \lambda_{n}(t)\right) .
$$

Consider the system

$$
x(t)-V(t) x(t-\tau)=f(t)
$$

where we have equivalence to

$$
y(t)-E_{\lambda}(t) y(t-\tau)=\widetilde{f}(t),
$$

where $\tilde{f}(t)=U(t) f(t), y(t)=U(t) x(t)$. On the other hand, a component of the vector in system (2.1) is

$$
y_{i}(t)-\lambda_{i}(t) y_{i}(t-\tau)=\widetilde{f}_{i}(t), \quad i \in I_{n} .
$$


From Lemma 2.1, we have

$$
y_{i}(t)= \begin{cases}\widetilde{f}_{i}(t)+\sum_{j=1}^{\infty} \prod_{k=1}^{j} \lambda_{i}(t-(k-1) \tau) \tilde{f}_{i}(t-j \tau), & \lambda_{i, L}<1, \\ -\frac{\widetilde{f}_{i}(t+\tau)}{\lambda_{i}(t+\tau)}-\sum_{j=1}^{\infty} \prod_{k=1}^{j+1} \frac{1}{\lambda_{i}(t+k \tau)} \widetilde{f}_{i}(t+j \tau+\tau), & \lambda_{i, l}>1 .\end{cases}
$$

Thus, $\mathcal{A}^{-1}$ exists and

$$
\mathcal{A}^{-1}: C_{T} \rightarrow C_{T}, \quad \mathcal{A}^{-1} f(t)=x(t)=U^{T}(t) y(t), \quad t \in[0, T] .
$$

When $\lambda_{i, L}<1$, by (2.2) we get

$$
\left|y_{i}(t)\right| \leq \frac{\max _{t \in[0, T]}\left|\tilde{f}_{i}(t)\right|}{1-\lambda_{i, L}}, \quad i \in I_{i}
$$

i.e.,

$$
\max _{t \in[0, T]}\left|y_{i}(t)\right| \leq \frac{\max _{t \in[0, T]}\left|\tilde{f}_{i}(t)\right|}{1-\lambda_{i, L}}, \quad i \in I_{i}
$$

Thus, by (2.3) we have

$$
\begin{aligned}
\left|\mathcal{A}^{-1} f\right|_{0} & =\max _{t \in[0, T]}\left|U^{T}(t) y(t)\right|=\max _{t \in[0, T]}|y(t)|=\max _{t \in[0, T]}\left(\sum_{i=1}^{n} y_{i}^{2}(t)\right)^{\frac{1}{2}} \\
& \leq\left(\sum_{i=1}^{n} \max _{t \in[0, T]} y_{i}^{2}(t)\right)^{\frac{1}{2}} \leq\left(\sum_{i=1}^{n} \frac{\max _{t \in[0, T]}\left[\left.\widetilde{f}_{i}(t)\right|^{2}\right.}{\left(1-\lambda_{i, L}\right)^{2}}\right)^{\frac{1}{2}} \\
& \leq\left.\left(\sum_{i=1}^{n} \frac{1}{\left(1-\lambda_{i, L}\right)^{2}}\right)^{\frac{1}{2}} \tilde{f}\right|_{0}=\left(\sum_{i=1}^{n} \frac{1}{\left(1-\lambda_{i, L}\right)^{2}}\right)^{\frac{1}{2}}|U f|_{0} \\
& =\left(\sum_{i=1}^{n} \frac{1}{\left(1-\lambda_{i, L}\right)^{2}}\right)^{\frac{1}{2}}|f|_{0} .
\end{aligned}
$$

Obviously,

$$
\int_{0}^{T}\left|\mathcal{A}^{-1} f(t)\right| d t \leq\left(\sum_{i=1}^{n} \frac{1}{\left(1-\lambda_{i, L}\right)^{2}}\right)^{\frac{1}{2}} \int_{0}^{T}|f(t)| d t
$$

(2) Similar to the above proof, when $\lambda_{i, l}>1$, we get

$$
\begin{aligned}
& \left|\mathcal{A}^{-1} f\right|_{0} \leq\left(\sum_{i=1}^{n} \frac{1}{\left(1-\lambda_{i, l}\right)^{2}}\right)^{\frac{1}{2}}|f|_{0}, \\
& \int_{0}^{T}\left|\mathcal{A}^{-1} f(t)\right| d t \leq\left(\sum_{i=1}^{n} \frac{1}{\left(1-\lambda_{i, l}\right)^{2}}\right)^{\frac{1}{2}} \int_{0}^{T}|f(t)| d t .
\end{aligned}
$$

Let $X$ and $Y$ be two Banach spaces and let $L: D(L) \subset X \rightarrow Y$ be a linear operator, a Fredholm operator with index zero (meaning that $\operatorname{Im} L$ is closed in $Y$ and $\operatorname{dim} \operatorname{Ker} L=$ 
$\operatorname{codim} \operatorname{Im} L<+\infty)$. If $L$ is a Fredholm operator with index zero, then there exist continuous projectors $P: X \rightarrow X, Q: Y \rightarrow Y$ such that $\operatorname{Im} P=\operatorname{Ker} L, \operatorname{Im} L=\operatorname{Ker} Q=\operatorname{Im}(I-Q)$, and $L_{D(L) \cap K e r} P:(I-P) X \rightarrow \operatorname{Im} L$ is invertible. Denote by $K_{p}$ the inverse of $L_{P}$.

Let $\Omega$ be an open bounded subset of $X$, a map $N: \bar{\Omega} \rightarrow Y$ is said to be L-compact in $\bar{\Omega}$ if $Q N(\bar{\Omega})$ is bounded and the operator $K_{p}(I-Q) N(\bar{\Omega})$ is relatively compact. We first give the famous Mawhin continuation theorem.

Lemma 2.3 [23] Suppose that $X$ and $Y$ are two Banach spaces and $L: D(L) \subset X \rightarrow Y$ is a Fredholm operator with index zero. Furthermore, $\Omega \subset X$ is an open bounded set and $N: \bar{\Omega} \rightarrow Y$ is L-compact on $\bar{\Omega}$. If all the following conditions hold:

(1) $L x \neq \lambda N x, \forall x \in \partial \Omega \cap D(L), \forall \lambda \in(0,1)$,

(2) $N x \notin \operatorname{Im} L, \forall x \in \partial \Omega \cap \operatorname{Ker} L$,

(3) $\operatorname{deg}\{Q N, \Omega \cap \operatorname{Ker} L, 0\} \neq 0$,

then the equation $L x=N x$ has a solution on $\bar{\Omega} \cap D(L)$.

\section{Main results}

Theorem 3.1 Suppose that $\int_{0}^{T} p(t) d t=0, \varphi(t)$ is a nonzero periodic solution of (3.1) and there exists a constant $M>0$ such that

$\left(\mathrm{H}_{1}\right) \forall i \in I_{n}, \frac{\partial G}{\partial x_{i}}$ is bounded in the set $\triangle_{1}\left(\right.$ or $\left.\triangle_{2}\right)$, where

$$
\begin{aligned}
& \triangle_{1}=\left\{x: x=\left(x_{1}, x_{2}, \ldots, x_{n}\right) \in \mathbb{R}^{n}, x_{i} \in(-\infty, M], x_{j} \in \mathbb{R}, j \in I_{n}-\{i\}\right\}, \\
& \triangle_{2}=\left\{x: x=\left(x_{1}, x_{2}, \ldots, x_{n}\right) \in \mathbb{R}^{n}, x_{i} \in[-M, \infty), x_{j} \in \mathbb{R}, j \in I_{n}-\{i\}\right\} .
\end{aligned}
$$

$\left(\mathrm{H}_{2}\right) x_{i} \frac{\partial G}{\partial x_{i}}>0($ or $<0)$, whenever $\left|x_{i}\right|>M, i \in I_{n}$.

$\left(\mathrm{H}_{3}\right)$ Suppose that $\mu_{1}, \mu_{2}, \ldots, \mu_{n}$ are eigenvalues of $\frac{\partial^{2} F(v)}{\partial x^{2}}, v \in \mathbb{R}^{n}$, and there exists a constant $\lambda_{F} \geq 0$ such that

$$
\max \left\{\left|\mu_{1}\right|,\left|\mu_{2}\right|, \ldots,\left|\mu_{n}\right|\right\} \leq \lambda_{F} .
$$

Then system (1.1) has at least one T-periodic solution, if $\lambda_{0, i}<\frac{1}{2}\left(\right.$ or $\left.\sigma_{0, i}>1\right),\left(\lambda_{2, i} T n+\right.$ $\left.n \lambda_{1, i} \sqrt{n}\right) T+\lambda_{0, i}<1$, and $\tau=m T, m \in \mathbb{Z}$, where

$$
\begin{array}{ll}
\lambda_{0, i}=\max _{t \in[0, T]}\left\{\left|c_{i}(t)\right|, i \in I_{n}\right\}, & \lambda_{1, i}=\max _{t \in[0, T]}\left\{\left|c_{i}^{\prime}(t)\right|, i \in I_{n}\right\}, \\
\lambda_{2, i}=\max _{t \in[0, T]}\left\{\left|c_{i}^{\prime \prime}(t)\right|, i \in I_{n}\right\}, & \sigma_{0, i}=\min _{t \in[0, T]}\left\{\left|c_{i}(t)\right|, i \in I_{n}\right\} .
\end{array}
$$

Proof Define

$$
\begin{aligned}
& A: C_{T} \rightarrow C_{T}, \quad[A x](t)=x(t)-C(t) x(t-\tau), \quad \forall t \in \mathbb{R}, \\
& N: C_{T}^{1} \rightarrow C_{T}, \quad(N x)(t)=-\frac{d}{d t} \operatorname{grad} F(x(t))-\operatorname{grad} G(x(t-\gamma(t)))+p(t), \\
& L: D(L) \subset C_{T} \rightarrow C_{T}^{1}, \quad L x=(A x)^{\prime \prime},
\end{aligned}
$$


where $D(L)=\left\{x: x \in C_{T}^{1}, x^{\prime \prime} \in C\left(\mathbb{R}, \mathbb{R}^{n}\right)\right\}$. Then system (1.1) obeys the operator equation $L x=N x$. We have $(x(t)-C(t) x(t-\tau))^{\prime \prime}=0, \forall x \in \operatorname{Ker} L$. Then

$$
x(t)-C(t) x(t-\tau)=\widetilde{c}_{1} t+\widetilde{c}_{2},
$$

where $\widetilde{c}_{1}, \widetilde{c}_{2} \in \mathbb{R}^{n}$. Since $x(t)-C(t) x(t-\tau) \in C_{T}$, we have $\widetilde{c}_{1}=0$. Let $\varphi(t) \in C\left(\mathbb{R}, \mathbb{R}^{n}\right)$ be a nonzero periodic solution of

$$
x(t)-C(t) x(t-\tau)=I,
$$

then $|\varphi(t)|^{2}>0$ and $\int_{0}^{T} \varphi^{2}(t) d t \neq 0$, where $I$ is an unit matrix. We get

$$
\operatorname{Ker} L=\left\{a_{0} \varphi(t): a_{0} \in \mathbb{R}\right\}, \quad \operatorname{Im} L=\left\{y: y \in C_{T}, \int_{0}^{T} y(s) d s=0\right\} .
$$

Obviously, $\operatorname{Im} L$ is closed in $C_{T}$ and $\operatorname{dim} \operatorname{Ker} L=\operatorname{codim} \operatorname{Im} L=n$. So $L$ is a Fredholm operator with index zero. Define continuous projectors $P, Q$ :

$$
P: C_{T} \rightarrow \operatorname{Ker} L, \quad(P x)(t)=\frac{\int_{0}^{T} x(t) \varphi(t) d t}{\int_{0}^{T} \varphi^{2} d t} \varphi(t)
$$

and

$$
Q: C_{T} \rightarrow C_{T} / \operatorname{Im} L, \quad Q y=\frac{1}{T} \int_{0}^{T} y(s) d s
$$

Let

$$
L_{P}=\left.L\right|_{D(L) \cap \operatorname{Ker} P}: D(L) \cap \operatorname{Ker} P \rightarrow \operatorname{Im} L,
$$

then

$$
L_{P}^{-1}=K_{P}: \operatorname{Im} L \rightarrow D(L) \cap \operatorname{Ker} P .
$$

Since $\operatorname{Im} L \subset C_{T}$ and $D(L) \cap \operatorname{Ker} P \subset C_{T}^{1}, K_{P}$ is an embedding operator. Hence $K_{P}$ is a completely continuous operator in $\operatorname{Im} L$. By the definitions of $Q$ and $N$, one knows that $Q N(\bar{\Omega})$ is bounded on $\bar{\Omega}$. Hence the nonlinear operator $N$ is L-compact on $\bar{\Omega}$. We complete the proof by three steps.

Step 1. Let $\Omega_{1}=\left\{x \in D(L) \subset C_{T}^{1}: L x=\lambda N x, \lambda \in(0,1)\right\}$. We show that $\Omega_{1}$ is a bounded set. We have $L x=\lambda N x \forall x \in \Omega_{1}$, i.e.,

$$
\frac{d^{2}}{d t^{2}}(x(t)-C(t) x(t-\tau))+\lambda \frac{d}{d t} \operatorname{grad} F(x(t))+\lambda \operatorname{grad} G(x(t-\gamma(t)))=\lambda p(t) .
$$

Integrating both sides of (3.2) over [0,T], we have

$$
\int_{0}^{T} \operatorname{grad} G(x(t-\gamma(t))) d t=0
$$


i.e., $\forall i \in I_{n}$,

$$
\int_{0}^{T} \frac{\partial G(x(t-\gamma(t)))}{\partial x_{i}} d t=0
$$

Let $\frac{\partial G}{\partial x_{i}}$ be bounded in $\triangle_{1}$ and

$$
x_{i} \frac{\partial G}{\partial x_{i}}>0, \quad \text { whenever }\left|x_{i}\right|>M
$$

Let

$$
E_{1}=\{t: t \in[0, T], x(t-\gamma(t)) \leq M\}, \quad E_{2}=\{t: t \in[0, T], x(t-\gamma(t))>M\} .
$$

By assumption $\left(\mathrm{H}_{1}\right)$, if $x_{i} \leq M$, there exists a constant $M_{1}>0$ such that $\left|\frac{\partial G}{\partial x_{i}}\right| \leq M_{1}$. From (3.3) and (3.4), we get

$$
\int_{E_{2}}\left|\frac{\partial G(x(t-\gamma(t)))}{\partial x_{i}}\right| d t=\int_{E_{2}} \frac{\partial G(x(t-\gamma(t)))}{\partial x_{i}} d t \leq \int_{E_{1}}\left|\frac{\partial G(x(t-\gamma(t)))}{\partial x_{i}}\right| d t \leq M_{1} T .
$$

Thus

$$
\int_{0}^{T}\left|\frac{\partial G(x(t-\gamma(t)))}{\partial x_{i}}\right| d t=\int_{E_{1}}\left|\frac{\partial G(x(t-\gamma(t)))}{\partial x_{i}}\right| d t+\int_{E_{2}}\left|\frac{\partial G(x(t-\gamma(t)))}{\partial x_{i}}\right| d t \leq 2 M_{1} T,
$$

i.e.,

$$
\begin{aligned}
\int_{0}^{T}|\operatorname{grad} G(x(t-\gamma(t)))| d t & =\int_{0}^{T}\left[\sum_{i=1}^{n}\left(\frac{\partial G(x(t-\gamma(t)))}{\partial x_{i}}\right)^{2}\right]^{\frac{1}{2}} d t \\
& \leq \int_{0}^{T}\left[\sum_{i=1}^{n}\left|\frac{\partial G(x(t-\gamma(t)))}{\partial x_{i}}\right|\right] d t \\
& \leq 2 n M_{1} T .
\end{aligned}
$$

We claim that there exists a point $t_{1} \in \mathbb{R}$ such that

$$
\left|x_{i}\left(t_{1}\right)\right| \leq M
$$

In fact, for $\forall t \in[0, T]$, we have $x_{i}(t-\gamma(t))>M$, and by (3.4), we have $\int_{0}^{T} \frac{\partial G(x(t-\gamma(t)))}{\partial x_{i}} d t>0$, which is a contradiction; see (3.3). So there must be a point $\xi \in[0, T]$ such that

$$
x_{i}(\xi-\lambda(\xi)) \leq M
$$

Similar to the above proof, there must be a point $\eta \in[0, T]$ such that

$$
x_{i}(\eta-\gamma(\eta)) \geq-M
$$


(1) If $x_{i}(\xi-\gamma(\xi)) \geq-M$, by (3.7) we have

$$
\left|x_{i}(\xi-\lambda(\xi))\right| \leq M
$$

Let $t_{1}=\xi-\gamma(\xi)$. This proves (3.6).

(2) If $x_{i}(\xi-\gamma(\xi))<-M$, from (3.8) and the fact that $x_{i}(t)$ is continuous on $\mathbb{R}$, there is a point $t_{1}$ between $\xi-\gamma(\xi)$ and $\eta-\gamma(\eta)$ such that $\left|x_{i}\left(t_{1}\right)\right| \leq M$. This also proves (3.6). Let $t_{1}=k \pi+t_{2}, k \in \mathbb{Z}, t_{2} \in[0, T]$. Then $\left|x_{i}\left(t_{1}\right)\right|=\left|x_{i}\left(t_{2}\right)\right| \leq M$. Hence we get

$$
\begin{aligned}
& \left|x_{i}(t)\right|=\max _{t \in[0, T]}\left|x_{i}\left(t_{2}\right)+\int_{t_{2}}^{t} x_{i}^{\prime}(s) d s\right| \leq\left|x_{i}\left(t_{2}\right)\right|+\int_{0}^{T}\left|x_{i}^{\prime}(s)\right| d s \leq M+\int_{0}^{T}\left|x^{\prime}(s)\right| d s, \\
& |x|_{0} \leq \sqrt{n}\left(M+\int_{0}^{T}\left|x^{\prime}(s)\right| d s\right) \leq \sqrt{n}\left(M+T^{\frac{1}{2}}\left(\int_{0}^{T}\left|x^{\prime}(s)\right|^{2} d s\right)^{\frac{1}{2}}\right) .
\end{aligned}
$$

Multiplying the two sides of system (3.2) by $x^{T}(t)$ and integrating them over $[0, T]$, combining with $\tau=m T$, by (3.9) we have

$$
\begin{aligned}
& -\int_{0}^{T}\left|x^{\prime}(t)\right|^{2} d t+\lambda_{2, i} \int_{0}^{T}|x(t)|^{2} d t+n \lambda_{1, i}|x|_{0} \int_{0}^{T}\left|x^{\prime}(t)\right| d t+\lambda_{0, i} \int_{0}^{T}\left|x^{\prime}(t)\right|^{2} d t \\
& \quad+\lambda \int_{0}^{T} x^{T}(t) \operatorname{grad} G\left(x(t-\gamma(t)) d t-\lambda \int_{0}^{T} x^{T}(t) p(t) d t \geq 0,\right.
\end{aligned}
$$

i.e.,

$$
\begin{aligned}
\left(1-\lambda_{0, i}\right) \int_{0}^{T}\left|x^{\prime}(t)\right|^{2} d t \leq & \lambda_{2, i} \operatorname{Tn}\left(M+\int_{0}^{T}\left|x^{\prime}(t)\right| d t\right)^{2} \\
& +n \lambda_{1, i} \sqrt{n}\left(M+\int_{0}^{T}\left|x^{\prime}(t)\right| d t\right) \int_{0}^{T}\left|x^{\prime}(t)\right| d t \\
& +\left(|p|_{0}+2 n M_{1}\right) T\left(M+\int_{0}^{T}\left|x^{\prime}(t)\right| d t\right) \\
= & \left.\lambda_{2, i} \operatorname{Tn}+n \lambda_{1, i} \sqrt{n}\right)\left(\int_{0}^{T}\left|x^{\prime}(t)\right| d t\right)^{2} \\
& +\left(2 \lambda_{2, i} \operatorname{Tn} M+n \lambda_{1, i} \sqrt{n} M+|p|_{0} T+2 n M_{1} T\right) \int_{0}^{T}\left|x^{\prime}(t)\right| d t \\
& +\lambda_{2, i} \operatorname{Tn} M^{2}+\left(|p|_{0}+2 n M_{1}\right) T M \\
\leq & \left(2 \lambda_{2, i} \operatorname{Tn}+n \lambda_{1, i} \sqrt{n}\right) T \int_{0}^{T}\left|x^{\prime}(t)\right|^{2} d t \\
& +\left(2 \lambda_{2, i} \operatorname{Tn} M+n \lambda_{1, i} \sqrt{n} M+|p|_{0} T+2 n M_{1} T\right) T^{\frac{1}{2}} \\
& \times\left(\int_{0}^{T}\left|x^{\prime}(t)\right|^{2} d t\right)^{\frac{1}{2}}+\lambda_{2, i} T n M^{2}+\left(|p|_{0}+2 n M_{1}\right) T M . \quad(3.9)
\end{aligned}
$$

From (3.9) and $\left(\lambda_{2, i} T n+n \lambda_{1, i} \sqrt{n}\right) T+\lambda_{0, i}<1$, there is a constant $M_{2}>0$ such that

$$
\int_{0}^{T}\left|x^{\prime}(t)\right|^{2} d t \leq M_{2}
$$


In view of (3.9) and (3.10), we get

$$
|x|_{0} \leq \sqrt{n}\left(M+T^{\frac{1}{2}} M_{2}^{\frac{1}{2}}\right):=M_{3}
$$

From Lemma 2.2, $(A x(t))^{\prime \prime}=A x^{\prime \prime}(t)-2 C^{\prime}(t) x^{\prime}(t-\tau)-C^{\prime \prime}(t) x(t-\tau)$ and (3.2), if $\lambda_{0, i}<\frac{1}{2}$, we have

$$
\begin{aligned}
x^{\prime \prime}(t)+A^{-1}\left[\lambda \frac{d}{d t}\right. & \operatorname{grad} F(x(t))+\lambda \operatorname{grad} G(x(t-\gamma(t)))] \\
=A^{-1}\left[2 C^{\prime}(t) x^{\prime}(t-\tau)+C^{\prime \prime}(t) x(t-\tau)+A^{-1}(\lambda p(t))\right] & \\
\int_{0}^{T}\left|x^{\prime \prime}(t)\right| d t \leq & \left(\sum_{i=1}^{n} \frac{1}{\left(1-\lambda_{0, i}\right)^{2}}\right)^{\frac{1}{2}} \\
& \times\left(\int_{0}^{T}\left|\frac{\partial^{2} F(x(t)}{\partial x^{2}}\right|\left|x^{\prime}(t)\right| d t+\int_{0}^{T}|\operatorname{grad} G(x(t-\gamma(t)))| d t\right. \\
& \left.+2 T \lambda_{1, i} \int_{0}^{T}\left|x^{\prime}(t)\right| d t+T \lambda_{2, i}|x|_{0}+T|p|_{0}\right) .
\end{aligned}
$$

From assumption $\left(\mathrm{H}_{3}\right)$ and (3.10)-(3.12), we get

$$
\begin{aligned}
& \int_{0}^{T}\left|x^{\prime \prime}(t)\right| d t \\
& \quad \leq\left(\sum_{i=1}^{n} \frac{1}{\left(1-\lambda_{0, i}\right)^{2}}\right)^{\frac{1}{2}}\left(\lambda_{F} T^{\frac{1}{2}} M_{2}^{\frac{1}{2}}+2 n M_{1} T+2 T \lambda_{1, i} T^{\frac{1}{2}} M_{2}^{\frac{1}{2}}+T \lambda_{2, i} M_{3}+T|p|_{0}\right) .
\end{aligned}
$$

So there exists a constant $M_{4}>0$ such that

$$
\int_{0}^{T}\left|x^{\prime \prime}(t)\right| d t \leq M_{4}
$$

Since $x(t) \in C_{T}^{1}, \int_{0}^{T} x^{\prime}(t) d t=0$, there is a constant vector $\alpha \in \mathbb{R}^{n}$ such that $x^{\prime}(\alpha)=0$; then by (3.13) we get

$$
\left|x^{\prime}(t)\right| \leq \int_{0}^{T}\left|x^{\prime \prime}(t)\right| d t \leq M_{4}
$$

Thus

$$
\left|x^{\prime}\right|_{0} \leq M_{4}
$$

Step 2. Let $\Omega\{x \in \operatorname{Ker} L: Q N x=0\}$, we shall prove that $\Omega_{2}$ is a bounded set. We have $x(t)=a_{0} \varphi(t), a_{0} \in \mathbb{R} \forall x \in \Omega_{2}$; then

$$
\int_{0}^{T} \operatorname{grad} G\left(a_{0} \varphi(t-\gamma(t))\right) d t=\int_{0}^{T} \operatorname{grad} G\left(a_{0} \varphi(t)\right) d t=0
$$


When $\lambda_{0, i}<\frac{1}{2}, i \in I_{n}$, we have

$$
\begin{aligned}
\varphi_{i}(t) & =A^{-1}(1)=1+\sum_{j=1}^{\infty} \prod_{k=1}^{j} c_{i}(t-(k-1) \tau) \\
& \geq 1-\sum_{j=1}^{\infty} \prod_{k=1}^{j} \lambda_{0, i}=1-\frac{\lambda_{0, i}}{1-\lambda_{0, i}} \\
& =\frac{1-2 \lambda_{0, i}}{1-\lambda_{0, i}}:=\delta>0 .
\end{aligned}
$$

Then we have

$$
|\varphi(t)| \geq \sqrt{n} \delta
$$

Thus

$$
a_{0} \leq \frac{M}{\sqrt{n} \delta}
$$

Otherwise, if, $\forall t \in[0, T],\left|a_{0} \varphi(t)\right|>M$, then from assumption $\left(\mathrm{H}_{2}\right)$, we have

$$
\frac{\partial G\left(a_{0} \varphi(t)\right)}{\partial x_{i}}>0 \quad(\text { or }<0), \quad i \in I_{n},
$$

which is a contradiction; see (3.14). When $\sigma_{0, i}>1, i \in I_{n}$, we have

$$
\begin{aligned}
\varphi_{i}(t) & =A^{-1}(1)=-\frac{1}{c_{i}(t+\tau)}-\sum_{j=1}^{\infty} \prod_{k=1}^{j} \frac{1}{c_{i}(t+k \tau)} \\
& \leq-\frac{1}{\lambda_{i, l}}-\sum_{j=1}^{\infty} \prod_{k=1}^{j+1} \frac{1}{\lambda_{0, i}} \\
& =-\frac{1}{\lambda_{i, l}-1}:=\gamma<0 .
\end{aligned}
$$

Then we have

$$
|\varphi(t)| \geq \sqrt{n}|\gamma|
$$

Thus

$$
a_{0} \leq \frac{M}{\sqrt{n}|\gamma|}
$$

Otherwise, if $\forall t \in[0, T],\left|a_{0} \varphi(t)\right|>M$, then from assumption $\left(\mathrm{H}_{2}\right)$, we have

$$
\frac{\partial G\left(a_{0} \varphi(t)\right)}{\partial x_{i}}>0 \quad(\text { or }<0), \quad i \in I_{n},
$$

which is a contradiction; see (3.14). Hence $\Omega_{2}$ is a bounded set. 
Step 3. Let $\Omega=\left\{x \in C_{T}^{1}:|x|_{0}<n M_{2}+1,\left|x^{\prime}\right|_{0}<n M_{4}+1\right\}$, then $\Omega_{1} \cup \Omega_{2} \subset \Omega, \forall(x, \lambda) \in$ $\partial \Omega \times(0,1)$, and from the above proof, $L x \neq \lambda N x$ is satisfied. Obviously, condition (2) of Lemma 2.3 is also satisfied. Now we prove that condition (3) of Lemma 2.3 is satisfied. We have $\left|x^{0}\right|=\left|a_{0} \varphi\right|_{0}, a_{0} \in \mathbb{R}, \forall x^{0} \in \partial \Omega \cap \operatorname{Ker} L$. There at least exists a $i \in I_{n}$ such that $\left|x_{i}^{0}\right|>M$. When $x_{i}^{0}>M$, take the homotopy

$$
H(x, \mu)=\mu x+(1-\mu) Q N x, \quad x \in \bar{\Omega} \cap \operatorname{Ker} L, \mu \in[0,1] .
$$

Then, by using assumption $\left(\mathrm{H}_{2}\right)$, we have $H(x, \mu) \neq 0$. When $x_{i}^{0}<-M$, take the homotopy

$$
H(x, \mu)=-\mu x-(1-\mu) Q N x, \quad x \in \bar{\Omega} \cap \operatorname{Ker} L, \mu \in[0,1] .
$$

We also have $H(x, \mu) \neq 0$. Then by degree theory,

$$
\begin{aligned}
\operatorname{deg}\{Q N, \Omega \cap \operatorname{Ker} L, 0\} & =\operatorname{deg}\{H(\cdot, 0), \Omega \cap \operatorname{ker} L, 0\} \\
& =\operatorname{deg}\{H(\cdot, 1), \Omega \cap \operatorname{ker} L, 0\} \\
& =\operatorname{deg}\{I, \Omega \cap \operatorname{ker} L, 0\} \neq 0 .
\end{aligned}
$$

Applying Lemma 2.3, we reach the conclusion.

Remark 3.1 When $\frac{1}{2} \leq \lambda_{0, i}<1$ or $\sigma_{0, i}<1$, there are no existence results for periodic solutions for system (1.1). We hope that there is interest in doing further research on this issue.

As an application, we consider the following system:

$$
\frac{d^{2}}{d t^{2}}(x(t)-C(t) x(t-4 \pi))+\frac{d}{d t} \operatorname{grad} F(x(t))+\operatorname{grad} G(x(t-5 \cos t))=p(t),
$$

where

$$
\begin{aligned}
& x(t)=\left(x_{1}(t), x_{2}(t)\right)^{T}, \quad \tau=4 \pi, \quad \gamma(t)=5 \cos t, \quad C(t)=\operatorname{diag}\left(\frac{\sin t}{1,000}, \frac{\cos t}{1,000}\right), \\
& F(x)=\frac{1}{2 \pi}\left(x_{1}^{2}+2 x_{1} x_{2}+x_{2}^{2}+2 x_{1}+3 x_{2}+1\right), \quad G(x)=\frac{1}{\sqrt{2} \pi}\left(x_{1}+x_{2}\right), \\
& p(t)=(\sin t, \cos t)^{T} .
\end{aligned}
$$

Clearly, system (3.15) is a particular case of system (1.1). Obviously,

$$
\operatorname{grad} G(x)=\frac{1}{\sqrt{2} \pi}\left(x_{1}, x_{2}\right)^{T}, \quad \frac{\partial^{2} F(v)}{\partial x^{2}}=\left(\begin{array}{cc}
\frac{1}{\pi} & \frac{1}{\pi} \\
\frac{1}{\pi} & \frac{1}{\pi}
\end{array}\right) .
$$

Here assumptions $\left(\mathrm{H}_{1}\right)-\left(\mathrm{H}_{2}\right)$ are all satisfied. In addition,

$$
\begin{aligned}
& T=2 \pi, \quad \lambda_{0, i}=\lambda_{1, i}=\lambda_{2, i}=\frac{1}{1,000}, \quad n=2, \\
& \left(\lambda_{2, i} T n+n \lambda_{1, i} \sqrt{n}\right) T+\lambda_{0, i} \approx 0.0976<1 .
\end{aligned}
$$


By using Theorem 3.1, when $\lambda_{0, i}<\frac{1}{2}$, we know that system (3.15) has at least one $2 \pi$ periodic solution.

\section{Competing interests}

The authors declare that they have no competing interests.

\section{Authors' contributions}

ZM performed all the steps of proof in this research and also wrote the paper. BD suggested many good ideas that made this paper possible and helped to improve the manuscript. All authors read and approved the final manuscript.

\section{Author details}

${ }^{1}$ Tianmu College, Zhejiang A and F University, Hangzhou, China. ${ }^{2}$ Department of Mathematics, Huaiyin Normal University, Huaian, China.

\section{Acknowledgements}

The authors wish to thank the anonymous referee for his/her valuable suggestions to this paper. This work is supported by the NSFC of China (11171085)

Received: 23 March 2014 Accepted: 3 June 2014 Published online: 23 September 2014

\section{References}

1. Kuang, Y: Delay Differential Equations with Applications in Population Dynamics. Academic Press, New York (1993)

2. Hale, J: Theory of Functional Differential Equations. Springer, New York (1997)

3. Kolmannovskii, $\mathrm{V}$, Myshkis, A: Introduction to the Theory and Applications of Functional Differential Equations. Kluwer Academic, London (1999)

4. Xu, S, Lam, J, Zou, Y: Further results on delay-dependent robust stability conditions of uncertain neutral systems. Int J. Robust Nonlinear Control 15, 233-246 (2005)

5. Babram, MA, Ezzinbi, K: Periodic solutions of functional differential equations of neutral type. J. Math. Anal. Appl. 204, 898-909 (1996)

6. Corduneanu, C: Existence of solutions for neutral functional differential equations with causal operators. J. Differ. Equ. 168, 93-101 (2000)

7. Weng, P, Liang, M: The existence and behavior of periodic solution of Hematopoiesis model. Math. Appl. 8, 434-439 (1995)

8. Chen, FD: Positive periodic solutions of neutral Lotka-Volterra system with feedback control. Appl. Math. Comput. $162,1279-1302$ (2005)

9. Beretta, E, Solimano, F, Takeuchi, Y: A mathematical model for drug administration by using the phagocytosis of red blood cell. J. Math. Biol. 35, 1-19 (1996)

10. Rabah, R, Sklyar, GM, Rezounenko, AV: Stability analysis of neutral type systems in Hilbert space. J. Differ. Equ. 214 391-428 (2005)

11. Shen, J, Liu, Y, Li, J: Asymptotic behavior of solutions of nonlinear neutral differential equations with impulses. J. Math. Anal. Appl. 332, 179-189 (2007)

12. Wang, K: Global attractivity of periodic solution for neutral functional differential system with multiple deviating arguments. Math. Methods Appl. Sci. 34, 1308-1316 (2011)

13. Fridman, E: New Lyapunov-Krasovskii functionals for stability of linear retarded and neutral type systems. Syst. Control Lett. 40, 1087-1092 (2004)

14. Han, Q: On robust stability of neutral systems with time-varying discrete delay and norm-bounded uncertainty. Automatica 42, 313-322 (2001)

15. He, Y, She, J, Liu, G: Delay-dependent robust stability criteria for uncertain neutral systems with mixed delay. Syst. Control Lett. 51, 57-65 (2004)

16. Lu, S, Ren, J, Ge, W: Problems of periodic solutions for a kind of second order neutral functional differential equation. Appl. Anal. 82, 411-426 (2003)

17. Lu, S: On the existence of positive periodic solutions for neutral functional differential equation with multiple deviating arguments. J. Math. Anal. Appl. 280, 321-333 (2003)

18. Lu, S, Ge, W: Existence of positive periodic solutions for neutral logarithmic population model with multiple delays. J. Comput. Appl. Math. 166, 371-383 (2004)

19. Serra, E: Periodic solutions for some nonlinear differential equations of neutral type. Nonlinear Anal. 17, 139-151 (1991)

20. Liu, B, Huang, L: Existence and uniqueness of periodic solutions for a kind of first order neutral functional differential equation. J. Math. Anal. Appl. 322, 121-132 (2006)

21. Lu, S, Ge, W: On the existence of periodic solutions for a kind of second order $n$-dimensional neutral functional differential systems. Acta Math. Sin. Engl. Ser. 46, 601-610 (2003)

22. Du, B, Ge, W, Lu, S: Periodic solutions for generalized Liénard neutral equation with variable parameter. Nonlinear Anal. 70, 2387-2394 (2009)

23. Gaines, R, Mawhin, J: Coincidence Degree and Nonlinear Differential Equations. Springer, Berlin (1977)

doi:10.1186/s13661-014-0151-1

Cite this article as: $\mathrm{He}$ and $\mathrm{Du}$ : Periodic solutions for a kind of neutral functional differential systems. Boundary Value Problems 2014 2014:151. 